\title{
Efficient framework for predicting MiRNA-disease associations based on improved hybrid collaborative filtering
}

\author{
Ru Nie ${ }^{1,2+}$, Zhengwei Li ${ }^{1,2,3,4^{*}+}$ (D), Zhu-hong You ${ }^{*}$, Wenzheng Bao ${ }^{6}$ and Jiashu Li $i^{1,2}$
}

From Fifteenth International Conference on Intelligent Computing (ICIC 2019) Nanchang, China. 3-6 August 2019

\begin{abstract}
Background: Accumulating studies indicates that microRNAs (miRNAs) play vital roles in the process of development and progression of many human complex diseases. However, traditional biochemical experimental methods for identifying disease-related miRNAs cost large amount of time, manpower, material and financial resources.

Methods: In this study, we developed a framework named hybrid collaborative filtering for miRNA-disease association prediction (HCFMDA) by integrating heterogeneous data, e.g., miRNA functional similarity, disease semantic similarity, known miRNA-disease association networks, and Gaussian kernel similarity of miRNAs and diseases. To capture the intrinsic interaction patterns embedded in the sparse association matrix, we prioritized the predictive score by fusing three types of information: similar disease associations, similar miRNA associations, and similar disease-miRNA associations. Meanwhile, singular value decomposition was adopted to reduce the impact of noise and accelerate predictive speed.

Results: We then validated HCFMDA with leave-one-out cross-validation (LOOCV) and two types of case studies. In the LOOCV, we achieved 0.8379 of AUC (area under the curve). To evaluate the performance of HCFMDA on real diseases, we further implemented the first type of case validation over three important human diseases: Colon Neoplasms, Esophageal Neoplasms and Prostate Neoplasms. As a result, 44, 46 and 44 out of the top 50 predicted disease-related miRNAs were confirmed by experimental evidence. Moreover, the second type of case validation on Breast Neoplasms indicates that HCFMDA could also be applied to predict potential miRNAs towards those diseases without any known associated miRNA.
\end{abstract}

Conclusions: The satisfactory prediction performance demonstrates that our model could serve as a reliable tool to guide the following research for identifying candidate miRNAs associated with human diseases.

*Correspondence: zwli@cumt.edu.cn; zhuhongyou@gmail.com

${ }^{\dagger}$ Ru Nie and Zhengwei Li have contributed equally to this work

${ }^{1}$ Engineering Research Center of Mine Digitalization of Ministry

of Education, China University of Mining and Technology, Xuzhou 221116,

China

${ }^{5}$ School of Computer Science, Northwestern Polytechnical University,

Xi'an 710072, China

Full list of author information is available at the end of the article

(c) The Author(s) 2021. Open Access This article is licensed under a Creative Commons Attribution 4.0 International License, which permits use, sharing, adaptation, distribution and reproduction in any medium or format, as long as you give appropriate credit to the original author(s) and the source, provide a link to the Creative Commons licence, and indicate if changes were made. The images or other third party material in this article are included in the article's Creative Commons licence, unless indicated otherwise in a credit line to the material. If material is not included in the article's Creative Commons licence and your intended use is not permitted by statutory regulation or exceeds the permitted use, you will need to obtain permission directly from the copyright holder. To view a copy of this licence, visit http://creativecommons.org/licenses/by/4.0/. The Creative Commons Public Domain Dedication waiver (http://creativecommons.org/publicdomain/zero/1.0/) applies to the data made available in this article, unless otherwise stated in a credit line to the data. 
Keywords: miRNA-disease association prediction, Hybrid collaborative filtering, Heterogeneous data, Singular value decomposition

\section{Introduction}

MicroRNAs (miRNAs) are endogenous small noncoding RNAs (19-22 nucleotides) that could regulate gene expression by base-pairing to partially complementary mRNAs [1]. Since the first miRNA, lin-4, was discovered by Lee et al. in 1993 [2], more than 38,000 miRNA sequences from 271 organisms have been accumulated to date [3]. Plenty of evidence indicates that miRNAs play critical roles in many fundamental and important biological processes, such as immune response, transcription, proliferation and differentiation [4]. The mutation and dysregulated expression of miRNAs may be connected with the development and progression of many diseases $[5,6]$. For instance, miR-155 downregulated target gene TP53INP1 whose expression was strongly reduced in pancreatic ductal adenocarcinoma development [7]. Besides, induction of endogenous miR-340 expression was capable to suppress tumor cell migration and invasion, whereas miR-340 knockdown led to breast cancer cell migration and invasion [8]. Moreover, three most upregulated miRNAs (miR-221, 222, and 146) distinguished unequivocally between papillary thyroid carcinoma and normal thyroid [9]. Therefore, exploring the relationships between miRNAs and diseases could not only provide novel insights into disease pathogenesis at the molecular level, but also benefit the design of specific molecular tools for disease diagnosis, treatment and prevention [10, $11]$.

However, traditional in vivo or biochemical experiment for identifying disease-related miRNA candidates have multiple bottlenecks, such as long operation time, extremely high cost and false positive results [12, 13]. Consequently, quickly and automatically identifying these associations with in silico methods is a useful supplement for future experimental validation and could substantially reduce the cost and effort [14-16]. Actually, based on the generally accepted assumption that functionally similar miRNAs are likely to be associated with phenotypically similar diseases and vice versa, a large number of computational models have been proposed for identifying potential disease-related miRNAs in recent years. For example, Jiang et al. [17] explored a network-based computational model through hypergeometric distribution to prioritize disease-related miRNAs. Shi et al. [18] focused on the functional connections between miRNA targets and disease genes in proteinprotein interaction (PPI) networks and presented a novel method to identify disease-related miRNAs. Xu et al. [19] introduced an approach based on MTDN for prioritizing putative miRNAs associated with diseases by combining paired miRNA and mRNA expression data. In addition, Chen et al. [20] constructed HGIMDA framework to identify potential disease-related miRNAs by combing multiple source information, e.g., experimentally validated miRNA-disease relationship, disease semantic similarity, miRNA functional similarity, Gaussian interaction profile kernel similarity. Based on the same multiple source data, Chen et al. [21] also presented PRMDA to infer potential disease-related miRNAs by personalized recommendation-based algorithm. Although HGIMDA and PRMDA could be applied to those diseases without experimentally validated miRNA, the predictive accuracy needs to be further enhanced. In addition, Marissa et al. [22] presented an in-silico method named MAP for predicting putative miRNA-disease associations through network diffusion on multi-omics biological data including miRNA-gene associations, protein-protein interactions, and gene-disease associations, and so on. Yu et al. [23] proposed TCRWMDA for miRNA-disease association prediction through three-layer heterogeneous network combined with unbalanced random walk. The case study results indicate that TCRWMDA is an effective tool to predict the potential miRNA-disease associations. Li et al. [24]. developed a novel method named NIMCGCN which employs graph convolutional networks to extract feature representations and a neural inductive matrix completion model to generate association matrix completion. Experimental results indicate this method could be used for predicting those diseases without any known related miRNAs.

Collaborative filtering aims at predicting the user interest for a given item based on a collection of user profiles and there are already some basic applications in miRNA-disease associations (MDAs) prediction [25-27]. However, these approaches generally fail to achieve satisfactory results. In this work, we developed a computational approach named hybrid collaborative filtering for predicting miRNA-disease associations (HCFMDA) to infer putative associations between diseases and miRNAs. By fusing experimentally verified MDAs, disease similarity, and miRNA functional similarity to mine intrinsic discriminative information embedded in the correlations between diseases and miRNAs, HCFMDA could be applied for identifying 
potential miRNAs for those diseases without any known related miRNA. In the leave-one-out crossvalidation (LOOCV), HCFMDA achieved AUC (area under the curve) value of 0.8379 and demonstrated reliable predictive performance. In addition, we also used HCFMDA to carry out two types of case validation on four important human complex diseases (Colon Neoplasms, Esophageal Neoplasms, Prostate Neoplasms and Breast Neoplasms). As a result, 88\%, 92\%, 88\% and $92 \%$ out of the top 50 putative miRNAs for those 4 diseases were confirmed by experiment evidence. All the results indicate HCFMDA is effective and reliable for the prediction of MDAs.

\section{Materials and methods}

\section{Human miRNA-disease associations}

The experimentally verified human miRNA-disease associations for HCFMDA were retrieved from HMDD v2.0 database [28]. After the data preprocessing and verification, we obtained altogether 5430 experimentally validated associations between 383 diseases and 495 miRNAs. Then, we constructed an adjacency matrix $X \in \mathrm{R}^{N_{d} \times N_{m}}$ to represent the corresponding associations, where $N_{d}$ and $N_{m}$ are the number of the diseases and

$$
D_{d_{i}}\left(d_{j}\right)= \begin{cases}1, & \text { if } d_{j}=d_{i} \\ \max \left\{\rho * D_{d_{i}}\left(d_{j}^{\prime}\right) \mid d_{j}^{\prime} \in \text { children of } d_{j}\right\}, \text { otherwise }\end{cases}
$$

\section{MiRNA functional similarity}

Based on the hypothesis that miRNAs with similar functions tend to be related to similar disease phenotypes, Wang et al. pioneered the human miRNA functional similarity which is available at http://www.cuilab.cn/files/ images/cuilab/misim.zip [29]. We herein constructed matrix $F M$ to express their functional similarity scores, where the entity $F M_{i j}$ denotes the similarity of miRNA pair $\left\langle m_{i}, m_{j}\right\rangle$.

\section{Disease semantic similarity}

According to previous study [30], we introduced directed acyclic graph (DAG) to express disease based on the Medical Subject Headings (MeSH) descriptors of category $\mathrm{C}$ from http://www.nlm.nih.gov/. Disease $d_{i}$ could be denoted as $D A G\left(d_{i}\right)=\left(V\left(d_{i}\right), E\left(d_{i}\right)\right)$, where $V\left(d_{i}\right)$ is a set consisting all ancestral nodes of $d_{i}$ and $d_{i}$ itself and $E\left(d_{i}\right)$ represents all directed edges from parent nodes to their respective children. The semantic value of disease $d_{i}$ is defined by

$$
\operatorname{DSV}\left(d_{i}\right)=\sum_{d_{j} \in V\left(d_{i}\right)} D_{d_{i}}\left(d_{j}\right)
$$

where $D_{d_{i}}\left(d_{j}\right)$, as the semantic contribution value of disease $d_{j}$ to $d_{i}$, could be calculated as
miRNAs, respectively. Here, element $\mathrm{X}_{i j}$ in the matrix is 1 or 0 , with 1 representing a known association between disease $d_{i}$ and miRNA $m_{j}$, and 0 denoting an unknown one. Correspondingly, the matrix $X$ could be decomposed into row vectors:

$$
X=\left[r_{1}, \ldots, r_{N_{d}}\right]^{T}, r_{i}=\left[r_{i, 1}, \ldots, r_{i, N_{m}}\right], \quad i=1, \ldots, N_{d}
$$

where $T$ denotes transpose operation and row vector $r_{i}$ represents the interaction profile of disease $d_{i}$. As described below, this representation was mainly used for disease-based collaborative filtering. Alternatively, the matrix $X$ could also be decomposed into column vectors:

$$
X=\left[c_{1}, \ldots, c_{N_{m}}\right], c_{j}=\left[c_{j, 1}, \ldots, c_{j, N_{d}}\right]^{T}, \quad j=1, \ldots, N_{m}
$$

where column vector $c_{j}$ corresponds to miRNA $m_{j}$. Likewise, this representation could be used for miRNAbased collaborative filtering.

where $\rho$ is a contribution factor. The semantic contribution value of disease $d_{j}$ to $d_{i}$ is inversely proportional to the distance between them in the DAG.

Based on the idea that two diseases will be more similar if their DAGs overlaps more nodes, we constructed semantic similarly matrix $S D$ for those diseases. Each element of $S D$ denotes the semantic similarly of disease pair $\left\langle d_{k}, d_{l}\right\rangle$, which could be calculated as following:

$$
S D\left(d_{k}, d_{l}\right)=\frac{\sum_{t \in V\left(\mathrm{~d}_{k}\right) \cap V\left(\mathrm{~d}_{l}\right)}\left(D_{\mathrm{d}_{k}}(t)+D_{\mathrm{d}_{l}}(t)\right)}{\operatorname{DSV}\left(\mathrm{d}_{k}\right)+\operatorname{DSV}\left(\mathrm{d}_{l}\right)}
$$

\section{Gaussian kernel similarity for diseases and miRNAs}

Gaussian kernel similarity comes from the topological distribution of the experimentally verified MDAs. Herein, we introduced binary vector $B V\left(d_{i}\right)$ as the interaction profile for disease $d_{i}$, which is the $i t h$ row of the adjacent matrix $X$. Hence, Gaussian kernel similarity of disease pair $<d_{i}, d_{j}>$ could be expressed by 


$$
G D\left(d_{i}, d_{j}\right)=\exp \left(-\delta_{d} B V\left(d_{i}\right)-B V\left(d_{j}\right)\right)
$$

where $\delta_{d}$ is a parameter for adjusting kernel bandwidth, which could be generated through averaging the interaction profiles of all diseases.

$$
\delta_{d}=\frac{\delta_{d}^{\prime}}{\frac{1}{N_{d}} \sum_{i=1}^{N_{d}} B V\left(d_{i}\right)}
$$

In the same manner, Gaussian kernel similarity between miRNA pair $\left\langle m_{i}, m_{j}\right\rangle$ could be defined as follows:

$$
\begin{aligned}
& G M\left(m_{i}, m_{j}\right)=\exp \left(-\delta_{m} B V\left(m_{i}\right)-B V\left(m_{j}\right)\right) \\
& \delta_{m}=\frac{\delta_{m}^{\prime}}{\frac{1}{N_{m}} \sum_{i=1}^{N_{m}} B V\left(m_{i}\right)}
\end{aligned}
$$

where $B V\left(m_{i}\right)$ is the interaction profile for miRNA $m_{i}$ and $\delta_{m}$ is used to control kernel bandwidth.

\section{Integrated similarities for miRNAs and diseases}

Following the previous steps, the miRNA functional similarity, disease semantic similarity and Gaussian kernel similarity were generated separately. To cope with data sparsity and effectively utilize all kinds of similarities and correlations, we further constructed an integrated similarity matrix $I D$ for diseases and $I M$ for miRNAs respectively, which could be expressed as follows:

$$
\begin{aligned}
& I D\left(d_{i}, d_{j}\right)=\left\{\begin{array}{l}
\operatorname{SD}\left(d_{i}, d_{j}\right), \text { if } \operatorname{SSD}\left(d_{i}, d_{j}\right)>0 \\
G D\left(d_{i}, d_{j}\right), \text { otherwise }
\end{array}\right. \\
& I M\left(m_{i}, m_{j}\right)=\left\{\begin{array}{l}
F M\left(m_{i}, m_{j}\right), \text { if } \operatorname{FSM}\left(m_{i}, m_{j}\right)>0 \\
\operatorname{GM}\left(m_{i}, m_{j}\right), \text { otherwise }
\end{array}\right.
\end{aligned}
$$

To ensure the equal importance, each similarity value should be normalized to the same interval before integration. The specific approach is to subtract the mean value and divide it by the standard deviation of the corresponding matrix.

\section{HCFMDA}

In this work, by integrating heterogeneous data including the miRNA functional similarity, disease semantic similarity, known MDA networks, Gaussian kernel similarity of miRNAs and diseases, we proposed a pipeline named improved hybrid collaborative filtering to predict miRNA-disease associations (HCFMDA). Figure 1 illustrates the flowchart of the entire process of HCFMDA. In order to fully exploit highly discriminative feature information embedded in the sparse MDAs, we incorporated and fused three different association sources: different diseases associated with the same miRNA, different miRNAs associated with the same disease, and 'not-so-similar' diseases or miRNAs, which could make the model more robust to data sparsity.

Disease-based collaborative filtering predicted the score $\hat{x}_{i, j}$ of a test miRNA $m_{j}$ for a disease $d_{i}$ based on top- $N$ most similar diseases towards $d_{i}$. Consequently, the corresponding recommended score $\hat{x}_{i, j}$ could be represented by

$$
\left.\hat{x}_{i, j}=\frac{1}{N} \sum_{d_{u} \in T_{d}\left(d_{i}\right)} s_{d}\left(d_{i}, d_{u}\right) X_{u, j}, \mid T_{d}\left(d_{i}\right)\right) \mid=N
$$

where $T_{d}\left(d_{i}\right)$ and $s_{d}\left(d_{i}, d_{u}\right)$ represent a set of top- $N$ most similar diseases towards disease $d_{i}$ and the integrated similarity value of disease pair $\left\langle d_{i}, d_{u}\right\rangle$, respectively. It could be seen from Fig. $2 \mathrm{a}$ that this method only exploits the known associations between the test miRNA and similar diseases of $d_{i}$, which accounts for only a small part in the matrix. We denoted this predictive source as the set of similar disease associations (SDA):

$$
S D A_{i, j}=\left\{X_{u, j} \mid d_{u} \in T_{d}\left(d_{i}\right)\right\}
$$

Similarly, the predictive score $\hat{x}_{i, j}$ of test pair $\left\langle d_{i}, m_{j}\right\rangle$ of miRNA-based collaborative filtering could also be calculated by averaging the associations of similar miRNAs related to the same disease $d_{i}$.

$$
\left.\hat{x}_{i, j}=\frac{1}{N} \sum_{m_{v} \in T_{m}\left(m_{j}\right)} s_{m}\left(m_{j}, m_{v}\right) X_{i, v}, \mid T_{m}\left(m_{j}\right)\right) \mid=N
$$

where $T_{m}\left(m_{j}\right)$ and $s_{m}\left(m_{j}, m_{v}\right)$ denote a set of top- $N$ most similar miRNAs towards miRNA $m_{j}$ and the integrated similarity between miRNA $m_{j}$ and $m_{v}$, respectively. As illustrated in Fig. 2b, Eq. (15) only exploits the known similar miRNAs associated with the test disease for prediction. We refer to these predictive sources as the set of similar miRNA associations $(S M A)$ :

$$
S M A_{i, j}=\left\{X_{i, v} \mid m_{v} \in T_{m}\left(m_{j}\right)\right\}
$$

In practice, solely relying on such SDA or SMA is undesirable, particularly when the association adjacent matrix $X$ is very sparse. The predictive accuracy could be improved by incorporating more associations from those 'not-so-similar' diseases or miRNAs. As illustrated in Fig. 2c, those associations from 'not-so-similar' diseases or miRNAs could provide additional information to improve the prediction. In this work, we refer to this 


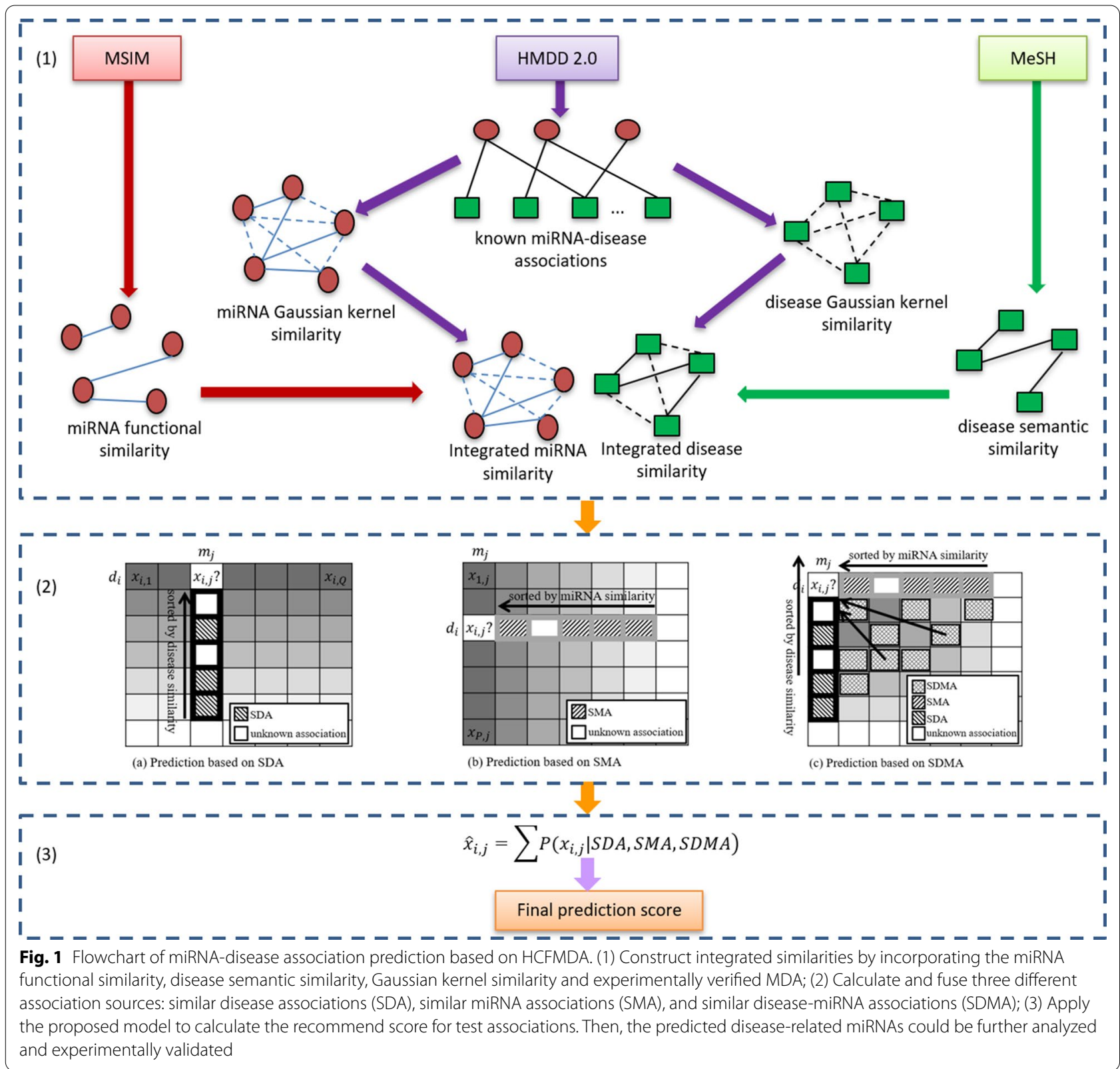

predictive source as similar disease-miRNA associations $(S D M A)$ :

$$
\begin{array}{r}
S D M A_{i, j}=\left\{X_{u, v} \mid d_{u} \in T_{d}\left(d_{i}\right), m_{v} \in T_{m}\left(m_{j}\right), u \neq i, v \neq j\right\} \\
(16) \\
\left.\hat{x}_{i, j}=\frac{1}{K} \sum_{X_{u, v} \in T_{d, m}\left(X_{i, j}\right)} s_{d, m}\left(X_{i, j}, X_{u, v}\right) X_{u, v}, \mid T_{d, m}\left(X_{i, j}\right)\right) \mid=K
\end{array}
$$

where $T_{d, m}\left(X_{i, j}\right)$ denotes a set of top- $K$ most similar miRNA-disease pairs. Here, we constructed $s_{d, m}\left(X_{i, j}, X_{u, v}\right)$ as the similarity between entity $X_{i, j}$ and $X_{u, v}$.

$$
s_{d, m}\left(X_{i, j}, X_{u, v}\right)=\frac{1}{\sqrt{\left(1 / s_{d}\left(d_{i}, d_{u}\right)\right)^{2}+\left(1 / s_{m}\left(m_{j}, m_{v}\right)\right)^{2}}}
$$

Each element of the matrix $X$ was employed as a separate predictor, whose confidence could be calculated according to its similarity towards the test association. We then predicted the expected value of the test association by averaging the individual predictions weighted by their confidence.

Finally, we calculated the expected value of the unknown test association $X_{i, j}$ by the following equation: 


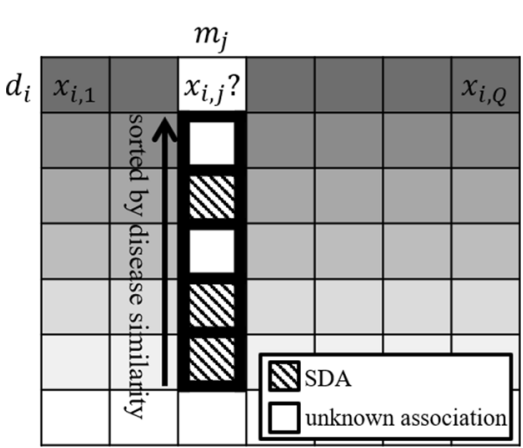

(a) Prediction based on SDA

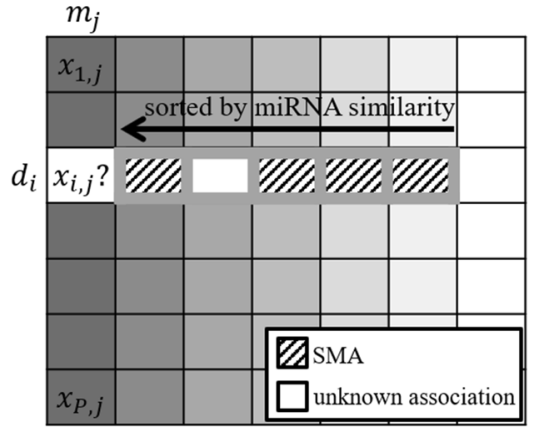

(b) Prediction based on SMA

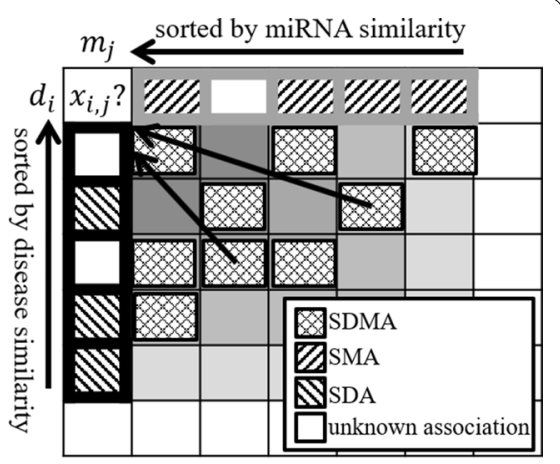

(c) Prediction based on SDMA

Fig. 2 Three different prediction methods based on SDA, SMA and SDMA

$$
\begin{aligned}
\hat{x}_{i, j}= & \sum P\left(X_{i, j} \mid S D A, S M A, S D M A\right) \\
= & \sum P\left(X_{i, j} \mid S D A\right) \alpha(1-\beta) \\
& +\sum P\left(X_{i, j} \mid S M A\right)(1-\alpha)(1-\beta) \\
& +\sum P\left(X_{i, j} \mid S D M A\right) \beta
\end{aligned}
$$

where $P\left(X_{i, j} \mid S D A, S M A, S D M A\right)$ denotes the estimating conditional probability depending on the predictors coming from the pool of $S D A, S M A$ and $S D M A$. Likewise, $P\left(X_{i, j} \mid S D A\right), P\left(X_{i, j} \mid S M A\right), P\left(X_{i, j} \mid S D M A\right)$ represent the pool of $S D A, S M A$ and $S D M A$ predictors, respectively. $\alpha$ and $\beta$ were used to control the selection (sampling) of data from those three different sources. If $\beta$ is equal 1 , HCFMDA only uses SDMA recommend score to predict potential miRNAs for given diseases. In addition, to remove noise and accelerate the operation speed, singular value decomposition (SVD) technique was applied in HCFMDA.

\section{Performance evaluation}

In practice, there are only 5430 experimentally verified MDAs (i.e., known associations) from HMDD V2.0 [28] and therefore most elements $(383 * 495-5430=184155)$ in adjacent matrix $X$ are zeros, which indicates that $X$ is very sparse and so it is not feasible to adopt multi-fold cross-validation to test the performance of our method. As demonstrated in a series of studies [31-37], leave-one-out cross-validation (LOOCV) is more rigorous and objective than independent dataset test and K-fold cross-validation. Therefore, we implemented LOOCV to validate the performance of HCFMDA. In the LOOCV, for a designated disease $d_{i}$, each known $d_{i}$-related association was left out in turn as a test sample and all other known associations (in total 5429) were used to train the model. Therefore, other miRNAs irrelevant to the disease $d_{i}$ along with the test miRNA were treated as candidate miRNAs. Then, we sorted all candidate miRNAs by the predictive scores derived from our model in descending order. If the rank of the test association exceeded a given threshold, we could view it as a successful identification. Receiver-operating characteristics (ROC) curve is a fundamental evaluation tool to illustrate diagnostic ability of a binary classifier. The ROC curve is generated by plotting true positive rate (TPR) against false positive rate (FPR) at different cutoff points. The corresponding formulas are as follows:

$$
\begin{aligned}
& T P R=\frac{T P}{T P+F N} \\
& F P R=\frac{F P}{F P+T N}
\end{aligned}
$$

where $T P, F N, F P$ and $T N$ represents true positive, false negative, false positive and true negative, respectively. More specifically, TP represents the number of known MDAs (positive samples) predicted correctly, and $F N$ is the number of positive samples that are falsely predicted to unknown MDAs (negative samples). Similarly, FP denotes the number of negative samples incorrectly predicted to positive samples while $T N$ stands for the number of negative samples predicted correctly.

In addition, we calculated AUC to evaluate the predictive performance of HCFMDA. The AUC is equivalent to the probability that a classifier will rank a randomly chosen positive sample higher than a randomly chosen negative one. Specifically, $\mathrm{AUC}=1$ represents a perfect test, while $\mathrm{AUC}=0.5$ means a worthless test. 


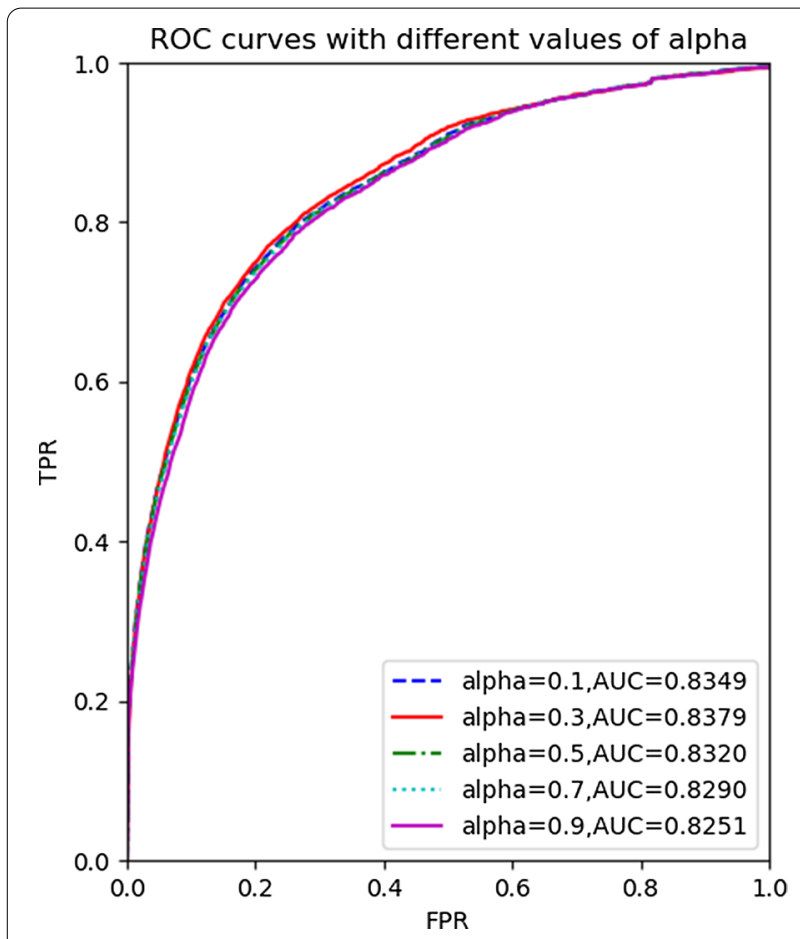

Fig. 3 Optimal search for proportion factor $\alpha$ performed by HCFMDA on HMDD v2.0 with $\beta=0.1$

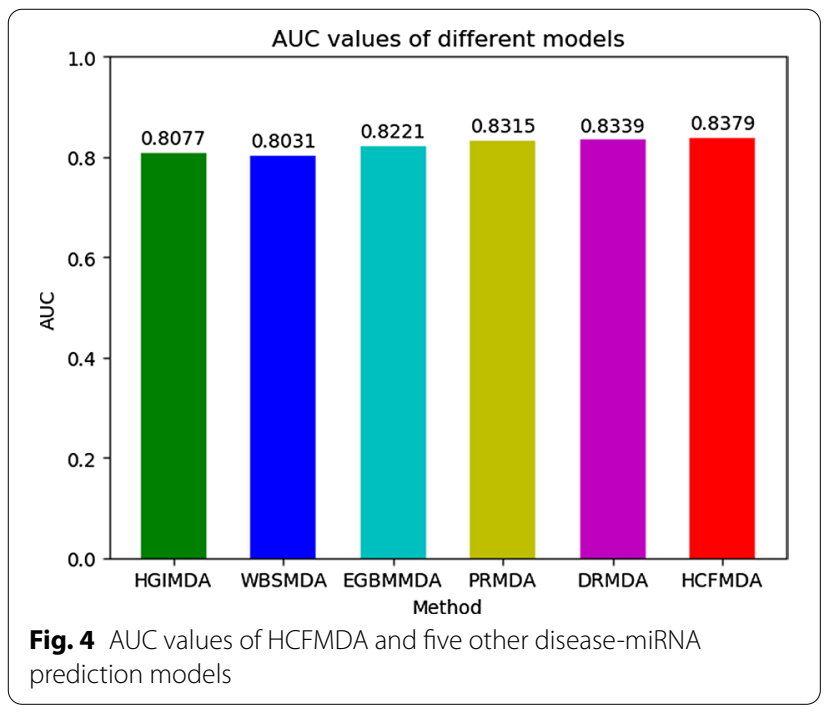

\section{Results}

\section{Experimental results performed by HCFMDA}

To verify the performance of HCFMDA to identify disease-related miRNAs, LOOCV was employed as testing strategy based on HMDD V2.0 dataset. For parameters $\alpha$ and $\beta$, we adopted grid search strategy to search their optimal values $(\alpha=0.3, \beta=0.1)$. Figure 3 illustrates the AUC values performed by HCFMDA on HMDD 2.0 by varying $\alpha$ from 0.1 to 1.0 with the step of 0.2 , where $\beta$ is set to 0.1 . It indicates that the fusion of multi-similarity measurement could enhance the prediction performance of HCFMDA model.

Then, we compared HCFMDA with other five stateof-the-art computational approaches: HGIMDA [20], WBSMDA [38], EGBMMDA [39], PRMDA [21] and DRMDA [40]. Figure 4 illustrates the AUC values comparison of those prediction models in the same framework of LOOCV. Our model achieved AUC of 0.8379, while the AUC values from HGIMDA, WBSMDA, EGBMMDA, PRMDA and DRMDA were 0.8077, $0.8031,0.8221,0.8315$ and 0.8339 , respectively. Because HCFMDA could capture high discriminative information embedded in the correlations between miRNAs and diseases, it achieved the superior predictive performance compared with the other five methods. Besides, the ROC curve of HCFMDA is smoother than those of other methods, which reflects HCFMDA is more robust and accurate. In addition, the time complexity of our model is obvious lower than the other methods. We attribute it to the introduction of SVD matrix decomposition that could reduce the impact of noise and improve the predictive speed. In conclusion, HCFMDA demonstrates reliable performance for predicting MDAs.

\section{Case validation 1: for diseases with known associated miRNAs}

Apart from the validation of LOOCV, we also carried out case studies for several important human diseases to further verify the performance of HCFMDA. Predictive results of HCFMDA were confirmed by matching experimentally verified MDAs (i.e., known associations) from two other independent databases: miR2Disease [41] and dbDEMC [42]. Herein, we adopted two types of case validations. At first, we applied HCFMDA over three different diseases, i.e. Colon Neoplasms $(\mathrm{CN})$, Esophageal Neoplasms (EN), and Prostate Neoplasms (PN). For a given disease, each time a known association in HMDD v2.0 was left out as a test sample and all unknown associations were taken as the candidate miRNAs, while all other known associations were used for training samples. In the second type of case validation for Breast Neoplasms (BN), we removed all known associations related to BN in HMDD v2.0 and then employed HCFMDA to predict potential BN-related miRNAs. The key point of this type of case validation is to ensure that the prioritization of putative miRNAs related to BN only makes use of the correlations of other phenotypic diseases similar to $\mathrm{BN}$. Therefore, it could be used to demonstrate whether our model is applicable to those isolated diseases without any known related miRNAs. 
As the most common type of gastrointestinal cancer, $\mathrm{CN}$ is the second-leading cause of cancer-related deaths in the USA. It was estimated that about 140,250 people was diagnosed with $\mathrm{CN}$, and 50,630 died from the disease in 2018 [43]. Since patients in early stage of CN have only mild symptoms and are hard to be detected, there is an urgent demand of novel diagnostic biomarkers for its early detection. Fortunately, a significant number of $\mathrm{CN}$-related miRNAs have been successfully identified in the past decades. For instance, Ma et al. [44] reported that the up-regulated miRNAs in $\mathrm{CN}$ including miR182, miR-17, miR-106a, miR-93, miR-200c, miR-92a, let-7a and miR-20a (FDR value $<5 \%$ ), while the downregulated miRNAs were miR-1195, miR-143 and miR-145 (FDR value $<5 \%$ ). Moreover, Marta et al. [45] identified and validated a signature of 6 miRNAs (miRNA19a, miRNA19b, miRNA15b, miRNA29a, miRNA335, and miRNA18a) as biomarkers that could differentiate significantly $\mathrm{CN}$ patients from those healthy people. In the case validation for $\mathrm{CN}$, we adopted HCFMDA to prioritize the top 50 miRNAs from candidate miRNAs (Table 1). We found that 9 out of the top 10 candidate miRNAs could be supported to be associated with $\mathrm{CN}$ by the experimental evidence. Besides, $88 \%$ of top 50 prioritized miRNAs were validated to be involved with $\mathrm{CN}$. For example, many experiments [46] reported that the expression level of has-mir-20a (ranked No.1 in Table 1) was significantly higher in $\mathrm{CN}$ tissue than those in the normal adjacent mucosa and suggests that it can be taken as a novel prognostic marker and therapeutic target for CN. Also, miR-18a (ranked No.2 in Table 1) was also confirmed by experiments and could play an important role in $\mathrm{CN}$ pathogenesis [47]. Actually, some of the rest unconfirmed miRNAs in Table 1 were also confirmed by recent studies. For example, hsa-mir-92 directly targeted the anti-apoptotic molecule BCL-2-interacting mediator of cell death (BIM) in colon cancer tissues and was recently proposed as a key oncogenic component of miR17-92 cluster through targeting and down-regulating the proapoptotic protein Bim in CN [48]. In addition, Antonio et al. [49] reported that has-miR-101 expression was differentially impaired in $\mathrm{CN}$ specimens and it might function as a tumor suppressor in $\mathrm{CN}$ and that its pharmacological restoration might hamper the aggressive behavior of CRC in vivo.

Esophageal neoplasms (EN), or esophageal cancer, occupies the sixth position among malignant tumors worldwide with regard to mortality and ranks fourth in China [50]. Due to lack of effective clinical diagnosis approaches for EN, it is often diagnosed at a more advanced stage and its overall 5-year survival rate is only about 25\% [51]. Therefore, investigating the mechanism of EN is seriously essential to improve its diagnosis,
Table 1 Predictive results of the top 50 prioritized miRNAs related to $\mathrm{CN}$ based on experimentally verified associations in HMDD v2.0 database

\begin{tabular}{|c|c|c|c|}
\hline miRNA & Evidence & miRNA & Evidence \\
\hline hsa-mir-20a & $\begin{array}{l}\text { dbDEMC, miR2D- } \\
\text { isease }\end{array}$ & hsa-mir-92a & Unconfirmed \\
\hline hsa-mir-18a & $\begin{array}{l}\text { dbDEMC, miR2D- } \\
\text { isease }\end{array}$ & hsa-mir-141 & $\begin{array}{l}\text { dbDEMC, } \\
\text { miR2Disease }\end{array}$ \\
\hline hsa-mir-19b & $\begin{array}{l}\text { dbDEMC, miR2D- } \\
\text { isease }\end{array}$ & hsa-mir-214 & $\mathrm{dbDEMC}$ \\
\hline hsa-mir-191 & $\begin{array}{l}\text { dbDEMC, miR2D- } \\
\text { isease }\end{array}$ & hsa-mir-30c & $\begin{array}{l}\text { dbDEMC, } \\
\text { miR2Disease }\end{array}$ \\
\hline hsa-mir-143 & $\begin{array}{l}\text { dbDEMC, miR2D- } \\
\text { isease }\end{array}$ & hsa-mir-93 & $\begin{array}{l}\text { dbDEMC, } \\
\text { miR2Disease }\end{array}$ \\
\hline hsa-mir-132 & miR2Disease & hsa-mir-34c & miR2Disease \\
\hline hsa-mir-29b & $\begin{array}{l}\text { dbDEMC, miR2D- } \\
\text { isease }\end{array}$ & hsa-mir-21 & $\begin{array}{l}\text { dbDEMC, } \\
\text { miR2Disease }\end{array}$ \\
\hline hsa-mir-19a & $\begin{array}{l}\text { dbDEMC, miR2D- } \\
\text { isease }\end{array}$ & hsa-mir-25 & $\begin{array}{l}\text { dbDEMC, } \\
\text { miR2Disease }\end{array}$ \\
\hline hsa-mir-34a & $\begin{array}{l}\text { dbDEMC, miR2D- } \\
\text { isease }\end{array}$ & hsa-mir-194 & $\begin{array}{l}\text { dbDEMC, } \\
\text { miR2Disease }\end{array}$ \\
\hline hsa-mir-101 & Unconfirmed & hsa-mir-32 & $\begin{array}{l}\text { dbDEMC, } \\
\text { miR2Disease }\end{array}$ \\
\hline hsa-let-7e & $\mathrm{dbDEMC}$ & hsa-mir-92b & Unconfirmed \\
\hline hsa-let-7d & $\mathrm{dbDEMC}$ & hsa-mir-205 & $\mathrm{dbDEMC}$ \\
\hline hsa-let-7a & $\begin{array}{l}\text { dbDEMC, miR2D- } \\
\text { isease }\end{array}$ & hsa-let-7 g & $\begin{array}{l}\text { dbDEMC, } \\
\text { miR2Disease }\end{array}$ \\
\hline hsa-mir-200b & $\mathrm{dbDEMC}$ & hsa-mir-222 & dbDEMC \\
\hline hsa-mir-127 & $\begin{array}{l}\text { dbDEMC, miR2D- } \\
\text { isease }\end{array}$ & hsa-mir-203 & $\begin{array}{l}\text { dbDEMC, } \\
\text { miR2Disease }\end{array}$ \\
\hline hsa-mir-125b & $\mathrm{dbDEMC}$ & hsa-mir-146a & $\mathrm{dbDEMC}$ \\
\hline hsa-mir-199a & Unconfirmed & hsa-mir-34b & $\begin{array}{l}\text { dbDEMC, } \\
\text { miR2Disease }\end{array}$ \\
\hline hsa-mir-223 & $\begin{array}{l}\text { dbDEMC, miR2D- } \\
\text { isease }\end{array}$ & hsa-mir-16 & dbDEMC \\
\hline hsa-let-7b & $\begin{array}{l}\text { dbDEMC, miR2D- } \\
\text { isease }\end{array}$ & hsa-mir-429 & dbDEMC \\
\hline hsa-mir-125a & $\begin{array}{l}\text { dbDEMC, miR2D- } \\
\text { isease }\end{array}$ & hsa-mir-221 & $\begin{array}{l}\text { dbDEMC, } \\
\text { miR2Disease }\end{array}$ \\
\hline hsa-mir-155 & $\begin{array}{l}\text { dbDEMC, miR2D- } \\
\text { isease }\end{array}$ & hsa-mir-200a & Unconfirmed \\
\hline hsa-let-7c & $\mathrm{dbDEMC}$ & hsa-mir-146b & Unconfirmed \\
\hline hsa-mir-106b & $\begin{array}{l}\text { dbDEMC, miR2D- } \\
\text { isease }\end{array}$ & hsa-mir-29a & $\begin{array}{l}\text { dbDEMC, } \\
\text { miR2Disease }\end{array}$ \\
\hline hsa-let-7f & $\begin{array}{l}\text { dbDEMC, miR2D- } \\
\text { isease }\end{array}$ & hsa-mir-95 & $\begin{array}{l}\text { dbDEMC, } \\
\text { miR2Disease }\end{array}$ \\
\hline hsa-let-7i & dbDEMC & hsa-mir-373 & dbDEMC \\
\hline
\end{tabular}

The column 1 and 3 list the top 1-25 and top 26-50 CN-related miRNAs, respectively

treatment and prognosis. Numerous recent studies have indicated that aberrant expression of miRNAs is involved in EN. For instance, Hu et al. [50] identified that miR-375 was downregulated in tumor tissue and cell line EC109 of EN samples when compared with normal tissues and cells. Experiments confirmed that as a tumor suppressor 
Table 2 Predictive results of the top 50 predicted miRNAs related to EN based on known associations in HMDD v2.0 database

\begin{tabular}{|c|c|c|c|}
\hline miRNA & Evidence & miRNA & Evidence \\
\hline hsa-mir-17 & $\mathrm{dbDEMC}$ & hsa-mir-24 & $\mathrm{dbDEMC}$ \\
\hline hsa-mir-18a & $\mathrm{dbDEMC}$ & hsa-mir-10b & $\mathrm{dbDEMC}$ \\
\hline hsa-mir-19b & dbDEMC & hsa-mir-30c & $\mathrm{dbDEMC}$ \\
\hline hsa-mir-125b & dbDEMC & hsa-mir-30a & dbDEMC \\
\hline hsa-mir-221 & dbDEMC & hsa-mir-181a & dbDEMC \\
\hline hsa-mir-16 & dbDEMC & hsa-mir-15b & dbDEMC \\
\hline hsa-mir-29a & $\mathrm{dbDEMC}$ & hsa-mir-93 & dbDEMC \\
\hline hsa-mir-200b & dbDEMC & hsa-mir-106a & $\mathrm{dbDEMC}$ \\
\hline hsa-mir-106b & dbDEMC & hsa-mir-18b & dbDEMC \\
\hline hsa-let-7d & dbDEMC & hsa-mir-132 & dbDEMC \\
\hline hsa-mir-1 & $\mathrm{dbDEMC}$ & hsa-mir-23b & $\mathrm{dbDEMC}$ \\
\hline hsa-let-7i & $\mathrm{dbDEMC}$ & hsa-mir-122 & unconfirmed \\
\hline hsa-let-7f & unconfirmed & hsa-mir-194 & dbDEMC,miR2Disease \\
\hline hsa-let-7e & dbDEMC & hsa-mir-7 & dbDEMC \\
\hline hsa-mir-222 & dbDEMC & hsa-mir-218 & unconfirmed \\
\hline hsa-mir-29b & dbDEMC & hsa-mir-127 & $\mathrm{dbDEMC}$ \\
\hline hsa-mir-429 & dbDEMC & hsa-mir-302c & dbDEMC \\
\hline hsa-mir-181b & dbDEMC & hsa-mir-199b & dbDEMC \\
\hline hsa-mir-142 & dbDEMC & hsa-mir-135a & dbDEMC \\
\hline hsa-mir-125a & dbDEMC & hsa-mir-193b & dbDEMC \\
\hline hsa-mir-182 & $\mathrm{dbDEMC}$ & hsa-mir-20b & dbDEMC \\
\hline hsa-let-7g & dbDEMC & hsa-mir-302b & $\mathrm{dbDEMC}$ \\
\hline hsa-mir-195 & dbDEMC & hsa-mir-107 & dbDEMC,miR2Disease \\
\hline hsa-mir-146b & dbDEMC & hsa-mir-204 & unconfirmed \\
\hline hsa-mir-9 & dbDEMC & hsa-mir-23a & dbDEMC \\
\hline
\end{tabular}

The column 1 and 3 list the top 1-25 and top 26-50 EN-related miRNAs, respectively

in EN cells, miR-375 inhibited cell proliferation and invasion by repressing the expression of its direct target $\mathrm{MTDH}$, an oncogene associated with tumorigenesis in EN. Herein, we took EN as a case validation and prioritized the candidate miRNAs of the disease. As illustrated in Table 2, all the top 10 predicted miRNAs associated with EN were successfully verified by experimental evidence collected from the two independent databases. Meanwhile, 46 out of the top 50 predicted miRNAs were also validated to be related to EN. For example, recent studies indicated that miR-195 was down-regulated in EN tissues compared with normal esophageal tissues $(P=0.05)$ and experimental results indicated that Cdc42 protein was reduced after miR-195 mimics transfected $(P=0.01)$ [52]. In addition, Zhang et al. [53] first presented that tanshinone IIA inhibited human EN cell growth through miR-122-mediated Pyruvate kinase M2 (PKM2) down-regulation pathway.

Prostate neoplasms (PN) is the most common malignancy and the third leading cancer-related cause of death
Table 3 Predictive results of the top 50 prioritized miRNAs related to PN based on known associations in HMDD v2.0 database

\begin{tabular}{|c|c|c|c|}
\hline miRNA & Evidence & miRNA & Evidence \\
\hline hsa-mir-21 & dbDEMC,miR2Disease & $\begin{array}{l}\text { hsa-mir- } \\
200 a\end{array}$ & dbDEMC \\
\hline hsa-mir-155 & $\mathrm{dbDEMC}$ & hsa-mir-23a & dbDEMC,miR2Disease \\
\hline hsa-let-7a & dbDEMC,miR2Disease & $\begin{array}{l}\text { hsa-mir- } \\
\text { 106b }\end{array}$ & dbDEMC \\
\hline $\begin{array}{c}\text { hsa-mir- } \\
146 a\end{array}$ & miR2Disease & hsa-mir-19b & dbDEMC,miR2Disease \\
\hline hsa-mir-17 & miR2Disease & hsa-mir-24 & dbDEMC,miR2Disease \\
\hline hsa-mir-20a & miR2Disease & hsa-let-7b & dbDEMC,miR2Disease \\
\hline hsa-mir-143 & dbDEMC,miR2Disease & hsa-mir-223 & dbDEMC,miR2Disease \\
\hline hsa-mir-18a & 24752237 & hsa-mir-34a & dbDEMC,miR2Disease \\
\hline hsa-let-7c & dbDEMC,miR2Disease & hsa-mir-15a & dbDEMC,miR2Disease \\
\hline hsa-mir-92a & 29568403 & hsa-let-7i & $\mathrm{dbDEMC}$ \\
\hline $\begin{array}{l}\text { hsa-mir- } \\
181 \mathrm{~b}\end{array}$ & dbDEMC,miR2Disease & $\begin{array}{l}\text { hsa-mir- } \\
\text { 200b }\end{array}$ & 24391862 \\
\hline hsa-let-7f & dbDEMC,miR2Disease & hsa-mir-25 & dbDEMC,miR2Disease \\
\hline hsa-mir-19a & $\mathrm{dbDEMC}$ & hsa-mir-142 & unconfirmed \\
\hline hsa-mir-1 & dbDEMC & hsa-mir-141 & miR2Disease \\
\hline hsa-mir-9 & $\mathrm{dbDEMC}$ & hsa-mir-222 & dbDEMC,miR2Disease \\
\hline hsa-mir-126 & dbDEMC,miR2Disease & hsa-let-7g & dbDEMC,miR2Disease \\
\hline hsa-let-7e & $\mathrm{dbDEMC}$ & hsa-mir-29c & $\mathrm{dbDEMC}$ \\
\hline hsa-mir-221 & dbDEMC,miR2Disease & $\begin{array}{c}\text { hsa-mir- } \\
125 a\end{array}$ & dbDEMC,miR2Disease \\
\hline hsa-let-7d & dbDEMC,miR2Disease & hsa-mir-203 & 21159887 \\
\hline hsa-mir-16 & dbDEMC,miR2Disease & $\begin{array}{c}\text { hsa-mir- } \\
106 a\end{array}$ & dbDEMC,miR2Disease \\
\hline hsa-mir-150 & dbDEMC & $\begin{array}{c}\text { hsa-mir- } \\
133 a\end{array}$ & $\mathrm{dbDEMC}$ \\
\hline hsa-mir-29a & dbDEMC,miR2Disease & hsa-mir-34b & dbDEMC \\
\hline hsa-mir-93 & 26124181 & hsa-mir-34c & $\mathrm{dbDEMC}$ \\
\hline hsa-mir-210 & miR2Disease & hsa-mir-27a & dbDEMC,miR2Disease \\
\hline $\begin{array}{l}\text { hsa-mir- } \\
200 c\end{array}$ & $\mathrm{dbDEMC}$ & hsa-mir-15b & $\mathrm{dbDEMC}$ \\
\hline
\end{tabular}

The column 1 and 3 list the top 1-25 and top 26-50 PN-related miRNAs, respectively. The evidences for the associations are either database studies or PMIDs of other experimental literatures

among men in the western world. Although the 5-year survival rate of $\mathrm{PN}$ is higher in early-stage after treatment with surgical resection or androgen deprivation therapy, one-third of treated PN patients will experience disease recurrence and progress into castration-resistant $\mathrm{PN}$, a more aggressive disease [54]. Therefore, an impressing need exists to identify novel miRNAs as tools or biomarkers for the prediction of aggressive PN. In the case validation for PN by HCFMDA, 8 of top 10 miRNAs and 44 out of top 50 candidate PN-associated miRNAs were validated by the two independent databases (see Table 3). Moreover, 4 of the rest 6 unsupported miRNAs 
Table 4 Comparison results of the first case study by HCFMDA and other five state-of-the art predictive models

\begin{tabular}{lllllll}
\hline Disease & HGIMDA & WBSMDA & EGBMMDA & PRMDA & DRMDA & HCFMDA \\
\hline Colon neoplasms & 45 & 45 & NULL & NULL & 46 & 44 \\
Esophageal neoplasms & 44 & 40 & 45 & 43 & NULL & 44 \\
Prostate neoplasms & 44 & 43 & 46 & 44
\end{tabular}

NULL denotes the corresponding model did not performed the case study for the designated disease

were verified by recent studies. For example, Williams et al. [55] identified miR-200b as a downstream target of androgen receptor and linked its expression to decreased tumorigenicity and metastatic capacity of the prostate cancer cells. In addition, as an "antimetastatic" miRNA in PN, miR-203 expression is specifically attenuated in bone metastatic prostate cancer suggesting a fundamental antimetastatic role for this miRNA [56].

Besides, we further made the comparison between HCFMDA and other five aforementioned MDA prediction models in terms of the case studies of diseases $\mathrm{CN}$, EN and PN. It could be seen from Table 4 that HCFMDA ranks second best among all predictive models for those three diseases. The predictive hit rate of HCFMDA is only lower than that of PRMDA in the case study of CN and EN, and that of EGBMMDA in the case study of PN, which fully demonstrates that HCFMDA could be used as a reliable tool for predicting disease-related miRNAs.

\section{Case validation 2: for diseases without known associated miRNAs}

To further validate the predictive performance of HCFMDA for those diseases without any known related miRNA, we also implemented another type of

Table 5 Predictive results of the top 50 prioritized miRNAs related to BN through removing all known BN-related miRNAs in HMDD V2.0 database

\begin{tabular}{|c|c|c|c|}
\hline miRNA & Evidence & miRNA & Evidence \\
\hline hsa-mir-367 & dbDEMC,HMDD & hsa-mir-608 & dbDEMC,HMDD \\
\hline hsa-mir-302c & dbDEMC,HMDD & hsa-mir-638 & dbDEMC,HMDD \\
\hline hsa-mir-302a & dbDEMC,HMDD & hsa-mir-518b & unconfirmed \\
\hline hsa-mir-302b & dbDEMC,HMDD & hsa-mir-602 & dbDEMC \\
\hline hsa-mir-488 & HMDD & hsa-mir-612 & dbDEMC \\
\hline hsa-mir-215 & dbDEMC,HMDD & hsa-mir-615 & dbDEMC \\
\hline hsa-mir-302d & dbDEMC,HMDD & hsa-mir-637 & dbDEMC \\
\hline hsa-mir-218 & $\mathrm{dbDEMC,HMDD}$ & hsa-mir-657 & dbDEMC \\
\hline hsa-mir-383 & $\mathrm{dbDEMC,HMDD}$ & hsa-mir-185 & dbDEMC \\
\hline hsa-let-7d & dbDEMC,miR2Disease,HMDD & hsa-mir-518c & dbDEMC \\
\hline hsa-let-7f & dbDEMC,miR2Disease,HMDD & hsa-mir-622 & dbDEMC \\
\hline hsa-let-7c & dbDEMC,HMDD & hsa-mir-583 & dbDEMC \\
\hline hsa-mir-19a & dbDEMC,HMDD & hsa-mir-557 & dbDEMC \\
\hline hsa-mir-153 & dbDEMC,HMDD & hsa-mir-600 & dbDEMC \\
\hline hsa-let-7b & $\mathrm{dbDEMC,HMDD}$ & hsa-mir-601 & $\mathrm{dbDEMC}$ \\
\hline hsa-let-7i & dbDEMC,miR2Disease,HMDD & hsa-mir-611 & unconfirmed \\
\hline hsa-mir-296 & dbDEMC,HMDD & hsa-mir-654 & dbDEMC \\
\hline hsa-let-7e & dbDEMC,HMDD & hsa-mir-662 & dbDEMC \\
\hline hsa-let-7a & dbDEMC,miR2Disease,HMDD & hsa-mir-769 & unconfirmed \\
\hline hsa-mir-429 & dbDEMC,miR2Disease,HMDD & hsa-mir-18a & dbDEMC,miR2Disease,HMDD \\
\hline hsa-mir-338 & dbDEMC,HMDD & hsa-mir-486 & dbDEMC,HMDD \\
\hline hsa-let-7g & dbDEMC,HMDD & hsa-mir-629 & dbDEMC,HMDD \\
\hline hsa-mir-20a & miR2Disease,HMDD & hsa-mir-596 & unconfirmed \\
\hline hsa-mir-19b & dbDEMC,HMDD & hsa-mir-17 & miR2Disease,HMDD \\
\hline hsa-mir-324 & HMDD & hsa-mir-339 & dbDEMC,HMDD \\
\hline
\end{tabular}

The column 1 and 3 list the top 1-25 and top 26-50 BN-related miRNAs, respectively 
case validation for Breast Neoplasms $(\mathrm{BN})$ by removing all the known BN-related associations in HMDD v2.0. That is to say, we only utilized the known associations of other diseases except $\mathrm{BN}$ and adopted the indirect way to predict $\mathrm{BN}$-related miRNAs. We then ranked all the 495 candidate miRNAs by their predictive scores and verified the top 50 ones according to the databases of dbDEMC, miR2Disease and HMDD v2.0. The predictive results (Table 5) indicated that all top 10 and 46 out of the top 50 prioritized miRNAs were confirmed by those databases. The achieved results indicate that HCFMDA could also be applied to predict novel miRNAs for those isolated diseases.

\section{Discussion}

Identification of novel disease-related miRNAs is beneficial for understanding disease pathogenesis at the molecular level, and developing effective disease diagnostic biomarkers and therapeutic tools. In this work, we proposed an efficient computational framework, HCFMDA, to improve the predictive performance of MDAs by integrating heterogeneous information: miRNA functional similarity, disease semantic similarity, known MDA networks, Gaussian kernel similarity of miRNAs and diseases. HCFMDA employs not only traditional disease-based and miRNA-based associations, but also associations from other 'not-so-similar' diseases and miRNAs to smooth the predictions. We then implemented LOOCV and two types of case validations over four important human cancers. The achieved results demonstrate that HCFMDA is indeed robust against data sparsity, which is better than other five state-of-the-art models, i.e., HGIMDA, WBSMDA, EGBMMDA, PRMDA, and DRMDA.

\section{Conclusions}

The excellent performance of HCFMDA mainly attributes to the following aspects. First, many kinds of heterogeneous data including miRNA functional similarity, disease semantic similarity, and known MDAs were integrated into our model, which contains highly discriminative information. Second, by fusing three kinds of similar associations including disease-based associations, miRNA-based associations, and other 'not-so-similar' diseases and miRNAs associations our model could fully mine and capture the intrinsic associations between miRNAs and diseases even if the MDA matrix is very sparse. Although some favorable results have been made, there still exists several limitations in HCFMDA. First, there are only 5430 known MDAs among 383 diseases and 495 miRNAs, and therefore the corresponding MDA matrix is very sparse and needs to be further enriched. Second, although we have integrated some heterogeneous data into our model, there is still room for improving the performance of HCFMDA by integrating more effective data sources which could provide more useful information for predicting MDAs. Moreover, we will improve the efficiency of our model by introducing graph-based recommendation filtering algorithms in the future.

\begin{abstract}
Abbreviations
MDA: MiRNA-disease association; HCF: Hybrid collaborative filtering; LOOCV: Leave-one-out cross-validation; BV: Binary vector; DAG: Directed acyclic graph; SDA: Similar disease associations; SMA: Similar miRNA associations; SDMA: Similar disease-miRNA associations; SVD: Singular value decomposition; ROC: Receiver operating characteristic; AUC: Area under curve; FPR: False positive rate; TPR: True positive rate; TP: True positive; FN: False negative; FP: False positive; TN: True negative.
\end{abstract}

\section{Acknowledgements}

We thank the editor and the anonymous reviewers for their comments and suggestions.

\section{About the supplement \\ This article has been published as part of BMC Medical Informatics and Deci- sion Making Volume 21 Supplement 1, 2021: Proceedings of the 2019 Interna- tional Conference on Intelligent Computing (ICIC 2019): medical informatics and decision making. The full contents of the supplement are available online at https://bmcmedinformdecismak.biomedcentral.com/articles/supplements/ volume-21-supplement-1.}

\section{Authors' contributions}

RN and ZL designed the study, prepared the data sets and wrote the manuscript. ZY, WB, and JL designed, performed and analyzed experiments. All authors have read and approved the final manuscript.

\section{Funding}

This work is supported by the Science and technology innovation 2030"new generation artificial intelligence" major project (No. 2018aa0100) and the National Natural Science Foundation of China (Nos. 61873270, 61732012, 61902337). The funders had no role in study design, data collection and analysis, decision to publish, or preparation of the manuscript. The authors would like to thank the anonymous reviewers for their constructive advices.

Availability of data and materials

The datasets used and/or analyzed during the current study are available at https://github.com/ivantsinghua/HCFMDA.

\section{Declarations}

Ethics approval and consent to participate Not applicable.

\section{Consent for publication}

Not applicable.

\section{Competing interests}

The authors declare that they have no competing interests.

\section{Author details}

${ }^{1}$ Engineering Research Center of Mine Digitalization of Ministry of Education, China University of Mining and Technology, Xuzhou 221116, China. ${ }^{2}$ School of Computer Science and Technology, China University of Mining and Technology, Xuzhou 221116, China. ${ }^{3}$ Institute of Machine Learning and Systems Biology, College of Electronics and Information Engineering, Tongji University, Shanghai 201804, China. ${ }^{4}$ KUNPAND Communications (Kunshan) Co.,

Ltd., Suzhou 215300, China. ${ }^{5}$ School of Computer Science, Northwestern 
Polytechnical University, Xi'an 710072, China. ${ }^{6}$ School of Information Engineering, Xuzhou University of Technology, Xuzhou 221018, China.

Received: 8 August 2021 Accepted: 23 August 2021

Published online: 30 August 2021

\section{References}

1. Bartel DP. MicroRNAs: genomics, biogenesis, mechanism, and function. Cell. 2004;116(2):281-97.

2. Lee RC, Feinbaum RL, Ambros V. The C. elegans heterochronic gene lin-4 encodes small RNAs with antisense complementarity to lin-14. Cell. 1993;75(5):843.

3. Kozomara A, Birgaoanu M, Griffiths-Jones S. miRBase: from microRNA sequences to function. Nucleic Acids Res. 2018;47(D1):D155-62.

4. Karp $X$, Ambros V. Encountering MicroRNAs in cell fate signaling. Science. 2005:310(5752):1288-9.

5. Zhao Q, Xie D, Liu H, Wang F, Yan GY, Chen X. SSCMDA: spy and super cluster strategy for MiRNA-disease association prediction. Oncotarget. 2018;9(2):1826-42.

6. Liu H, Ren G, Chen H, Liu Q, Yang Y, Zhao Q. Predicting IncRNA-miRNA interactions based on logistic matrix factorization with neighborhood regularized. Knowl Based Syst. 2020;191:105261.

7. Meritxell G, Mylène S, Min-Jue X, Carla C, Richard T, Julien G, et al. Tumor protein 53 -induced nuclear protein 1 expression is repressed by miR-155, and its restoration inhibits pancreatic tumor development. Proc Natl Acad Sci USA. 2007;104(41):16170-5.

8. Wu Z, Wu Q, Wang C, Wang X, Huang J, Zhao J, et al. miR-340 inhibition of breast cancer cell migration and invasion through targeting of oncoprotein c-Met. Cancer. 2011:117(13):2842-52.

9. He H, Jazdzewski K, Li W, Liyanarachchi S, Nagy R, Volinia S, et al. The role of microRNA genes in papillary thyroid carcinoma. Proc Natl Acad Sci USA. 2005;102(52):19075-80.

10. You ZH, Huang ZA, Zhu Z, Yan GY, Li ZW, Wen Z, et al. PBMDA: a novel and effective path-based computational model for miRNA-disease association prediction. PLoS Comput Biol. 2017;13(3):e1005455.

11. Li Z, Nie R, You Z, Cao C, Li J. Using discriminative vector machine model with 2DPCA to predict interactions among proteins. BMC Bioinform. 2019;20(Suppl 25):694.

12. Chen X, Xie D, Wang L, Zhao Q, Liu H. BNPMDA: Bipartite network projection for MiRNA—disease association prediction. Bioinformatics. 2018;34(18):3178-86.

13. Zhao $Q$, Yang $Y$, Ren $G$, Ge E, Fan C. Integrating bipartite network projection and KATZ measure to identify novel CircRNA-disease associations. IEEE Trans Nanobiosci. 2019;18(4):578-84.

14. Chen $X$, Yan CC, Zhang X, You ZHJBiB. Long non-coding RNAs and complex diseases: from experimental results to computational models. Brief Bioinform. 2017;18(4):515-39.

15. Hu H, Zhang L, Ai H, Zhang H, Fan Y, Zhao Q, et al. HLPI-Ensemble: prediction of human IncRNA-protein interactions based on ensemble strategy. RNA Biol. 2018;15(6):797-806.

16. Li J, Li Z, Nie R, You Z, Bao W. FCGCNMDA: predicting miRNA-disease associations by applying fully connected graph convolutional networks. Mol Genet Genom MGG. 2020;295(5):1197-209.

17. Jiang $Q$, Hao Y, Wang $G$, Juan $L$, Zhang $T$, Teng $M$, et al. Prioritization of disease microRNAs through a human phenome-microRNAome network. BMC Syst Biol. 2010;4(s1):S2.

18. Shi H, Xu J, Zhang G, Xu L, Li C, Wang L, et al. Walking the interactome to identify human miRNA-disease associations through the functional link between miRNA targets and disease genes. BMC Syst Biol. 2013;7(101):1752-2509.

19. Xu J, Li C, LV J, Li Y, Xiao Y, Shao T, et al. Prioritizing candidate disease miRNAs by topological features in the miRNA target-dysregulated network: case study of prostate cancer. Mol Cancer Ther. 2011;10(10):1857-66.

20. Chen $X$, Clarence YC, Zhang X, You ZH, Huang YA, Yan GY. HGIMDA: heterogeneous graph inference for miRNA-disease association prediction. Oncotarget. 2016;7(40):65257-69.
21. You ZH, Wang LP, Chen X, Zhang S, Li XF, Yan GY, et al. PRMDA: personalized recommendation-based MiRNA-disease association prediction. Oncotarget. 2017;8(49):85568-83.

22. Sumathipala M, Weiss ST. Predicting miRNA-based disease-disease relationships through network diffusion on multi-omics biological data. Sci Rep. 2020;10(1):8705.

23. Yu L, Shen X, Zhong D, Yang J. Three-layer heterogeneous network combined with unbalanced random walk for miRNA-disease association prediction. Front Genet. 2019;10:1316.

24. Li J, Zhang S, Liu T, Ning C, Zhang Z, Zhou W. Neural inductive matrix completion with graph convolutional networks for miRNA-disease association prediction. Bioinformatics. 2020;36(8):2538-46.

25. Wang J, de Vries A, Reinders M (eds). Unifying user-based and item-based collaborative filtering approaches by similarity fusion. In: The 29th annual international ACM SIGIR conference on research and development in information retrieva; 2006 August 6-11; New York: ACM Press; 2006.

26. Li ZS, Liu B, Yan C. CFMDA: collaborative filtering-based MiRNA-disease association prediction. Multimed Tools Appl. 2017;7006:1-14.

27. Gu C, Bo L, Li X, Cai L, Chen H, Li K, et al. Network-based collaborative filtering recommendation model for inferring novel disease-related miRNAs. RSC Adv. 2017;7(71):44961-71.

28. Li Y, Qiu C, Tu J, Geng B, Yang J, Jiang T, et al. HMDD v2.0: a database for experimentally supported human microRNA and disease associations. Nucleic Acids Res. 2014;42(Database issue):D1070-4.

29. Wang D, Wang J, Lu M, Song F, Cui Q. Inferring the human microRNA functional similarity and functional network based on microRNA-associated diseases. Bioinformatics. 2010;26(13):1644-50.

30. Xuan P, Han K, Guo M, Guo Y, Li J, Ding J, et al. Prediction of microRNAs associated with human diseases based on weighted $k$ most similar neighbors. PLoS ONE. 2013;8(8):e70204.

31. Basith S, Manavalan B, Shin TH, Lee G. SDM6A: a web-based integrative machine-learning framework for predicting $6 \mathrm{~mA}$ sites in the rice genome. Mol Ther Nucleic Acids. 2019;18:131-41.

32. Manavalan B, Basith S, Shin TH, Wei L, Lee G. Meta-4mCpred: a sequencebased meta-predictor for accurate DNA $4 \mathrm{mC}$ site prediction using effective feature representation. Mol Ther Nucleic Acids. 2019;16:733-44.

33. Boopathi V, Subramaniyam S, Malik A, Lee G, Manavalan B, Yang DC. mACPpred: a support vector machine-based meta-predictor for identification of anticancer peptides. Int J Mol Sci. 2019;20(8):1964.

34. Manavalan B, Basith S, Shin TH, Wei L, Lee G. mAHTPred: a sequencebased meta-predictor for improving the prediction of anti-hypertensive peptides using effective feature representation. Bioinformatics. 2019;35(16):2757-65.

35. Basith S, Manavalan B, Shin TH, Lee G. iGHBP: Computational identification of growth hormone binding proteins from sequences using extremely randomised tree. Comput Struct Biotechnol J. 2018;16:412-20.

36. Manavalan B, Subramaniyam S, Shin TH, Kim MO, Lee G. Machinelearning-based prediction of cell-penetrating peptides and their uptake efficiency with improved accuracy. J Proteome Res. 2018;17(8):2715-26.

37. Manavalan B, Shin TH, Lee G. PVP-SVM: sequence-based prediction of phage virion proteins using a support vector machine. Front Microbiol. 2018:9:476.

38. Chen X, Yan CC, Zhang X, You ZH, Deng L, Liu Y, et al. WBSMDA: within and between score for MiRNA-disease association prediction. Sci Rep. 2016;6(1):21106.

39. Chen $X$, Huang L, Xie D, Zhao Q. EGBMMDA: extreme gradient boosting machine for MiRNA-disease association prediction. Cell Death Dis. 2018;9(1):3.

40. Chen $X$, Gong Y, Zhang DH, You ZH, Li ZW. DRMDA: deep representations-based miRNA-disease association prediction. J Cell Mol Med. 2018;22(1):472-85.

41. Jiang $Q$, Wang $Y$, Hao $Y$, Juan $L$, Teng $M$, Zhang $X$, et al. miR2Disease: a manually curated database for microRNA deregulation in human disease. Nucleic Acids Res. 2009;37(Database):D98-104.

42. Yang Z, Ren F, Liu C, He S, Sun G, Gao Q, et al. dbDEMC: a database of differentially expressed miRNAs in human cancers. BMC Genom. 2010;11(Suppl 4):S5.

43. Weinberg BA, Marshall JL. colon cancer in young adults: trends and their implications. Curr Oncol Rep. 2019;21(1):3. 
44. Qin M, Lie Y, Cun W, Yong-Yang Y, Bin Z, Zhong-Guang Z. Differential expression of colon cancer microRNA in microarry study. J Sichuan Univ. 2011;42(3):344-8.

45. Herreros Villanueva M, Duran S, Carmen Martín A, Pérez-Palacios R, VilaNavarro E, Marcuello M, et al. Plasma MicroRNA signature validation for early detection of colorectal cancer; 2019.

46. Zhang GJ, LiY, Zhou H, Xiao HX, Zhou T. miR20a is an independent prognostic factor in colorectal cancer and is involved in cell metastasis. Mol Med Rep. 2014;10(1):283-91.

47. Anna Brunet V, Carles P, Irene M, Anna F, Emma D, Aleydis P, et al. microRNA expression profile in stage III colorectal cancer: circulating miR-18a and miR-29a as promising biomarkers. Oncol Rep. 2013;30(1):320-6.

48. Schee K, Boye K, Abrahamsen TW, Fodstad $\varnothing$, Flatmark K. Clinical relevance of microRNA miR-21, miR-31, miR-92a, miR-101, miR-106a and miR-145 in colorectal cancer. BMC Cancer. 2012;12(1):505.

49. Antonio S, Maria Chiara V, Pasquale S, Cinzia C, Annamaria S, Laura V, et al. Loss of miR-101 expression promotes Wnt/ $\beta$-catenin signalling pathway activation and malignancy in colon cancer cells. J Pathol. 2013;229(3):379-89.

50. Hu C, Lv L, Peng J, Liu D, Wang X, Zhou Y, et al. MicroRNA-375 suppresses esophageal cancer cell growth and invasion by repressing metadherin expression. Oncol Lett. 2017;13(6):4769-75.
51. Guo Y, Chen Z, Zhang L, Zhou F, Shi S, Feng X, et al. Distinctive MicroRNA profiles relating to patient survival in esophageal squamous cell carcinoma. Can Res. 2008;68(1):26-33.

52. Fu M, Li S, Yu T, Qian L, Gao S, Hong Z, et al. The expression of miR-195 in esophageal cancer and its effect on proliferation of esophageal cancer cell lines. Acta Univ Med Nanjing; 2013.

53. Zhang H-S, Zhang F-J, Li H, Liu Y, Du G-Y, Huang Y-H. Tanshinone IIA inhibits human esophageal cancer cell growth through miR-122-mediated PKM2 down-regulation. Arch Biochem Biophys. 2016;598:50-6.

54. Xin Z, Jinfeng W. Prognostic role of microRNA-145 in prostate cancer: a systems review and meta-analysis. Prost Int. 2015;3(3):71-4.

55. Williams LTV, Dorina V, Elena V, Volpert OV. miR-200b inhibits prostate cancer EMT, growth and metastasis. PLoS ONE. 2013;8(12):e83991.

56. Saini S, Majid S, Yamamura S, Tabatabai L, Suh SO, Shahryari V, et al. Regulatory role of mir-203 in prostate cancer progression and metastasis. Clin Cancer Res. 2011;17(16):5287-98.

\section{Publisher's Note}

Springer Nature remains neutral with regard to jurisdictional claims in published maps and institutional affiliations.
Ready to submit your research? Choose BMC and benefit from:

- fast, convenient online submission

- thorough peer review by experienced researchers in your field

- rapid publication on acceptance

- support for research data, including large and complex data types

- gold Open Access which fosters wider collaboration and increased citations

- maximum visibility for your research: over $100 \mathrm{M}$ website views per year

At BMC, research is always in progress.

Learn more biomedcentral.com/submissions 